\title{
The effect of mild electrical stimulation with heat shock on diabetic KKAy mice
}

\author{
Yukari Kai ${ }^{1,2}$, Yu Tsurekawa ${ }^{1,2}$, Ryosuke Fukuda ${ }^{1}$, Kohei Omachi ${ }^{1,2}$, Mariam Piruzyan ${ }^{1}$, Yuka Okamoto ${ }^{1}$, Keishi Motomura ${ }^{1}$, Mary Ann \\ Suico $^{1}$, Tsuyoshi Shuto ${ }^{1}$, Tatsuya Kondo ${ }^{3}$, Eiichi Araki ${ }^{3}$ and Hirofumi Kai ${ }^{* 1}$ \\ ${ }^{1}$ Department of Molecular Medicine, Graduate School of Pharmaceutical Sciences, Kumamoto University, 5-1 Oe-honmachi, Chuo-ku, Kumamoto 862-0973, \\ Japan \\ ${ }^{2}$ Program for Leading Graduate Schools "HIGO (Health life science: Interdisciplinary and Glocal Oriented) Program", Kumamoto University, 5-1 Oe-honmachi, \\ Chuo-ku, Kumamoto 862-0973, Japan \\ ${ }^{3}$ Department of Metabolic Medicine, Faculty of Life Sciences, Kumamoto University, 1-1-1 Honjo, Chuo-ku, Kumamoto 860-8556, Japan
}

\begin{abstract}
Diabetes mellitus (DM) is a growing health burden worldwide, and the number of patients with metabolic syndrome is increasing as well. The therapeutic strategies for DM and metabolic syndrome, and prevention of disease progression are therefore urgently needed. Insulin resistance is the main pathology in DM and metabolic syndrome. We previously showed that co-treatment of mild electrical stimulation (MES) with heat shock (HS) enhances insulin sensitivity in vitro and in diabetic mouse models. Moreover, MES+HS ameliorated the pathophysiology in human subjects with DM or metabolic syndrome in clinical trials. Despite the growing evidence that MES+HS could be a novel therapeutic approach, it is unclear whether long-term MES+HS treatment from the early stage of disease has preventive effect for DM. Thus, we assessed the effect of MES+HS on the pathophysiology of maturity-onset obesity using diabetic KKAy mice. Here, we showed that long-term treatment of MES+HS alleviated diabetic condition of KKAy mice. Hyperglycemia and insulin resistance were improved by MES+HS treatment. MES+HS also tended to suppress renal hypertrophy and increased the expression of podocyte-specific genes in the kidney. Collectively, our study suggests that long-term treatment of MES+HS is an effective and well-tolerated therapeutic strategy to decelerate the progression of DM and metabolic syndrome at least in part due to enhanced insulin sensitivity and podocyte function.
\end{abstract}

\begin{abstract}
Abbreviations: DM: diabetes mellitus, MES: mild electrical stimulation, HS: heat shock
\end{abstract}

\section{Introduction}

Type 2 diabetes mellitus (T2DM) is a serious health problem worldwide, and the number of patients with $\mathrm{T} 2 \mathrm{DM}$ is rapidly increasing [1]. About $80-90 \%$ of T2DM patients are diagnosed as obese. Obesity, caused by the accumulation of visceral fat, is the fundamental cause of the development of metabolic syndrome [2]. Chronic metabolic syndrome exhibits high-blood pressure, hyperglycemia and insulin resistance, leading to T2DM, arteriosclerosis and cardiovascular defects [3]. Especially, progression of T2DM leads to intercurrent diabetic retinopathy and diabetic nephropathy $[4,5]$. Therefore, treatment that can control the progression of metabolic syndrome and T2DM at the primary stage of these diseases is needed. Previously, we optimized the conditions of mild electrical stimulation (MES) that induce several physiological response signals and molecules such as the insulin receptor (IR)-Akt pathway, MKK3b/6b-p38MAPK, JNK, p53, AMPK and HSP72 [6-9]. Co-treatment with MES and $42^{\circ} \mathrm{C}$ of heat shock (HS) (MES+HS) ameliorated visceral obesity and diabetic pathophysiology in high-fat diet-induced and leptin receptor mutant $(\mathrm{db} / \mathrm{db})$ mice [6]. Moreover, MES+HS treatment reduced the metabolic abnormalities and inflammation in subjects with metabolic syndrome or T2DM in clinical trials $[10,11]$. The accumulation of IR in the lipid rafts within cell plasma membrane, followed by enhanced sensitivity to insulin, by MES+HS was suggested to contribute to the improvement of insulin resistance in T2DM [12]. Hence, MES+HS can be a useful treatment for metabolic syndrome and T2DM. However, it is unclear whether long-term treatment with MES+HS from the early stage of disease is tolerable and effective in preventing the development of maturityonset obesity and T2DM.

Here, we performed long-term treatment (28 weeks) with MES+HS on diabetic KKAy mice. KKAy is a well-established mouse model of metabolic syndrome with maturity-onset obesity, and spontaneously develops hyperglycemia, high blood pressure, and insulin resistance, which are quite similar to those observed in human T2DM $[13,14]$. The present study showed that long-term treatment with MES+HS decreased the blood glucose levels at the late stage of disease and also improved insulin resistance compared to sham-treated group. Additionally, long-term MES+HS treatment improved renal hypertrophy that is the hallmark of primary stage diabetic nephropathy and increased the expression of glomerular visceral epithelial cells and podocyte marker genes expression. This study revealed the beneficial

Correspondence to: Hirofumi Kai, Department of Molecular Medicine, Graduate School of Pharmaceutical Sciences, Kumamoto University, 5-1 Oe-honmachi, Chuo-ku, Kumamoto City 862-0973, Japan. Tel: +81-96-371-4405; Fax: +81-96371-4405; E-mail: hirokai@gpo.kumamoto-u.ac.jp

Key words: type 2 diabetes, insulin resistance, diabetic nephropathy, electrical stimulation, heat shock, KKAy mouse

Received: November 29, 2015; Accepted: December 15, 2015; Published: December 17, 2015 
effects and tolerability of long-term MES+HS treatment on metabolic syndrome and T2DM progression.

\section{Materials and methods}

\section{Mice and in vivo MES+HS treatment}

Seven-week-old male KK-Ay/taJcl (KKAy) mice (Charles River Laboratories, Inc., Kanagawa, Japan) were housed in an individual cage. KKAy mice were treated with MES+HS for 10 min twice a week as previously described [6]. Briefly, mice were placed in a well-ventilated chamber and MES was applied to unanesthetized mice through a pair of $10-\mathrm{cm}$ diameter electro-conductive and thermo-generative rubber electrodes, which are padded with moist soft cotton cloth. The electrodes were connected to Biometronome ${ }^{\mathrm{TM}}$ (Tsuchiya Gum Co., Ltd.) that delivered $5 \mathrm{~V}$ and 55 pulses per second (pps) of direct current with individual pulse duration of 0.1 -millisecond $(\mathrm{ms})$. The temperature of the electrodes was adjusted to $42^{\circ} \mathrm{C}$ for simultaneous heat shock treatment. All the animal experiments were approved by the Animal Care and Use Committee of Kumamoto University (\#25230E).

\section{Blood glucose measurement}

Blood samples were collected from tail vein of 15-hr fasted KKAy mice and used for the measurement of fasted blood glucose. Random fedblood glucose was measured by using blood obtained from adlibitum-fed mice. Fasted and random fed blood glucose levels were measured alternatively every week.

\section{Real-time Quantitative RT-PCR Analysis (RT-Q-PCR)}

Total RNA was isolated from renal tissue of KKAy mice with RNAiso Plus ${ }^{\oplus}$ (Takara Bio Inc.) following the manufacturer's instructions. Reverse transcription experiments and PCR amplifications were performed using PrimeScript ${ }^{\bullet}$ RT reagent kit and SYBR Premix Ex Taq ${ }^{\text {Tw }}$ (Takara Bio Inc.). The Q-PCR primers for mouse Podocin, Nephrin and Gapdh were reported previously [15]. The following primers were used for Q-PCR of mouse Synaptopodin: FW, 5'-GCCAGGGACCAGCCAGATA-3', and RV, 5'-AGGAGCCCAGGCCTTCTCT-3'.

\section{Insulin tolerance test (ITT)}

Insulin tolerance test was performed on 6 hour-fasted KKAy mice. Mice were given intraperitoneal injection of human regular insulin (1 unit/kg body weight; Novo Nordisk, Copenhagen, Denmark), and blood glucose level was assessed immediately before and at the indicated time points after injection.

\section{Measurement of mouse body and organ weight}

Mice were anesthetized and sacrificed after 28 weeks of treatment. Body weight was measured before final treatment and sacrifice. Visceral fat and kidneys were surgically collected immediately and washed with PBS. Organ weights were measured after removal of moisture from the organs.

\section{Statistical analysis}

Data are presented as mean \pm SE or SD. Significance of the difference between two groups was assessed with Student's unpaired two-tailed t-test. A $P$ value less than 0.05 is considered statistically significant.

\section{Results}

\section{MES+HS treatment ameliorated the diabetic phenotypes in KKAy mice}

To investigate the effect of MES+HS treatment on the pathological phenotypes in KKAy mice, 7-week-old KKAy mice were sham-treated (control; CON) or treated with MES+HS for 10 min twice a week for 28 weeks. The conditions of MES were $5 \mathrm{~V}, 0.1-\mathrm{ms}$ pulse width and 55 pps, and $\mathrm{HS}$ was at $42^{\circ} \mathrm{C}$. Mice did not show any pain or annoyance behavior as observed by their tendency to stay still during treatment. The diagram of experimental equipment of MES+HS treatment is shown in Figure 1A. Fasted and random fed blood glucose levels were determined every 2 weeks after the initiation of treatment. MES+HS had minimal effect on both fasted and random fed blood glucose levels within 20 weeks of treatment (Figure 1B and 1C). However, after 22 weeks, random fed blood glucose level was significantly decreased in MES+HS-treated KKAy mice (Figure 1D). Because MES+HS was previously shown to improve fat metabolism in high fat-fed and $\mathrm{db} / \mathrm{db}$ mice [6], we next determined whether MES+HS affects fat accumulation in KKAy mice. Although visceral fat (epididymal, mesenteric and retroperitoneal) weight was slightly suppressed by MES+HS compared with control, the difference was not statistically significant (Figure 1E). KKAy mice are known to have severe insulin resistance $[16,17]$ in addition to hyperglycemia. We thus performed insulin tolerance test (ITT) to examine the effect of MES+HS on insulin sensitivity. ITT was performed after 26 weeks of treatment. The result of ITT suggested that MES+HS improved insulin sensitivity in KKAy mice (Figure 1F). Collectively, these data indicated that MES+HS treatment ameliorated insulin resistance in these mice.

\section{MES+HS treatment ameliorated renal hypertrophy and} increased the mRNA expression of podocyte-specific genes

Nephropathy is one of the most common complications of diabetes, and diabetic nephropathy is the leading cause of end-stage renal disease. In type 1 diabetes patients, kidney is often enlarged even in the early stage of disease [18]. Renal hypertrophy is caused by various factors such as glomerular and tubular hypertrophy, mesangial cell expansion, cell proliferation, extracellular matrix accumulation, glomerular hypertension, and inflammatory cell infiltration $[19,20]$. Intriguingly, MES+HS treatment tended to suppress kidney weight in KKAy mice and it was not due to reduction of body weight (Figure 2A, B). In addition to hypertrophic signals, cell death signals are activated in glomerular visceral epithelial cells or podocytes with diabetic stress factors [21]. Podocytes are highly differentiated cells that are responsible for glomerular filtration system [22]. In diabetic nephropathy, the foot process effacement or detachment and apoptosis of podocytes are frequently observed $[20,23,24]$. Therefore, we investigated the effect of MES+HS treatment on podocytes. We determined the mRNA expressions of podocyte-specific genes, Synaptopodin, Podocin and Nephrin, which are the known markers of podocytes, in whole kidneys of KKAy mice. The expression of Synaptopodin mRNA was significantly up-regulated in MES+HS-treated group (Figure 2C). MES+HS also showed a trend of increase in Podocin and Nephrin mRNA levels in kidneys of KKAy mice (Figures 2D, 2E). These results suggested that MES+HS suppressed podocyte loss in KKAy mice kidney. Taken together, MES+HS suppressed renal hypertrophy and positively regulated podocyte-specific gene expression.

\section{Discussion}

Metabolic syndrome is promoted by crosstalk of systemic failure 


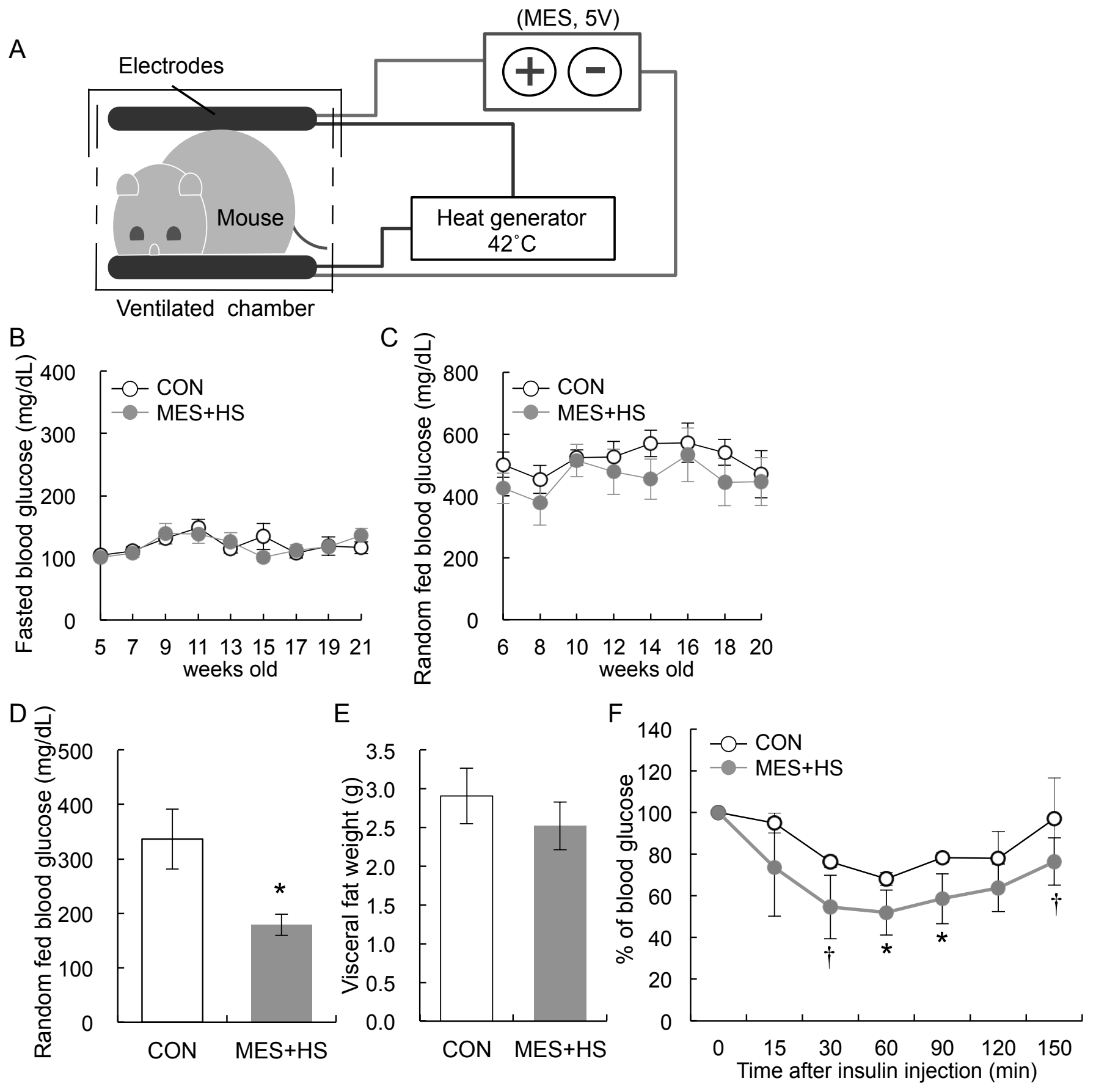

Figure 1. Effect of MES+HS treatment on diabetic KKAy mice

(a) Diagram of MES+HS treatment set-up in vivo. Seven-week-old KKAy mice were sham-treated (CON) or treated with MES+HS for 10 min twice a week for 28 weeks. Blood glucose levels of (b) 15 hour-fasted and (c) random fed KKAy mice were measured every 2 weeks during treatment. (d) Random fed blood glucose levels of KKAy mice sham-treated (CON) and treated with MES+HS were measured after 22 weeks of treatment. (e) Visceral fat weight of CON and MES+HS groups was measured after 28 weeks of treatment. (f) Insulin tolerance test was performed on mice at 26 weeks of treatment in 6-hr fasted state. Blood glucose levels were measured at the indicated time after insulin administration. Data are presented as mean (b-e) \pm S.E. or (f) \pm S.D. ( $\mathrm{n}=8$ for sham-treated control and $\mathrm{n}=5$ for MES + HS treated group). $\dagger \mathrm{P}<0.1,{ }^{*} \mathrm{P}<0.05$ vs. control group, assessed by unpaired $\mathrm{t}$-test.

such as hyperglycemia, insulin resistance, hypertension, glucose intolerance and dyslipidemia [3]. Chronic exposure to these risk factors leads to T2DM and other associated diseases. Especially, insulin resistance is thought to be a critical driving factor of T2DM development. Our previous study showed that MES+HS increased insulin sensitivity in T2DM mouse model [6]. Consistent with this report, the present study revealed that MES+HS treatment improves insulin resistance in KKAy mouse, a mouse model of metabolic syndrome with maturityonset obesity and T2DM (Figure 1E). We further found that long-term treatment of MES+HS in KKAy mice did not induce any adverse effects such as weight loss, hypoglycemia and inflammation (data not shown). These results are also consistent with the report showing that MES+HS 

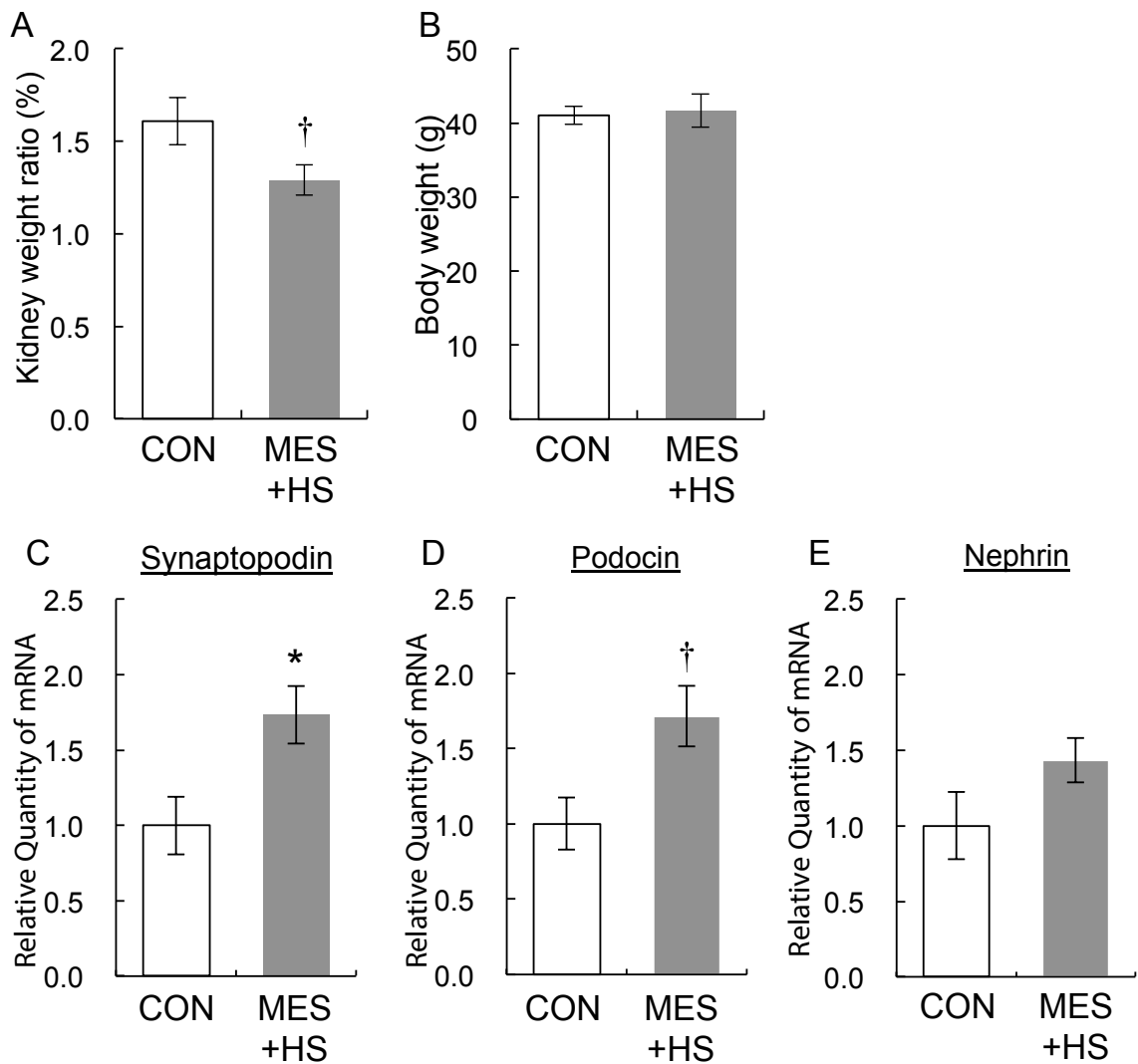

Figure 2. MES+HS decreased kidney weight and up-regulated podocyte-specific genes in KKAy mice.

(a) Mice were sacrificed after final treatment at 28 weeks of treatment, and kidney weights were determined. (b) Body weight was measured before the final treatment. (c-e) After the final treatment, total RNA was isolated from kidney of 28 weeks-MES+HS-treated and sham-treated (CON) KKAy mice. Q-PCR analysis was performed to assess the expression of the indicated podocyte-specific genes. Gapdh was used as internal control. Values are mean \pm S.E. ( $\mathrm{n}=3$ for sham-treated control and $\mathrm{n}=5$ for $\mathrm{MES}+\mathrm{HS}$ treated group). $\dagger \mathrm{P}<0.1, * \mathrm{P}<0.05$ vs. control group, assessed by unpaired t-test.

did not induce hypoglycemia or adversely affect food intake in normal mice and healthy human subjects $[6,25]$, which establishes the safety and tolerability of long-term MES+HS treatment.

Interestingly, MES+HS suppressed the renal hypertrophy in KKAy mice (Figure 2A). Renal hypertrophy is observed in the primary stage of diabetic nephropathy, and frequency of renal hypertrophy corresponds to the risk of developing end stage renal disease [18]. Hence, this result suggests that MES+HS has a preventive effect on diabetic nephropathy that is sequential to T2DM. Additionally, we showed that MES+HS treatment increased the mRNA expression of podocyte-associated genes in the kidneys of KKAy mouse (Figures 2C-2E). Podocytes are responsible for initiation and progression of renal injury induced by diabetic condition [26]. Diabetic conditions such as hyperglycemia, high blood pressure in glomeruli, insulin resistance and oxidative stress primarily induce podocyte foot process effacement, detachment from glomerular basement membrane and apoptosis [20]. Recently, it was reported that podocyte-specific loss of insulin signaling recapitulated the characteristics of diabetic nephropathy, and that insulin signaling is essential for the actin cytoskeleton organization of podocyte foot process to maintain the glomerular filtration barrier system [27]. Therefore, suppression of podocyte disorders through the activation of podocyte insulin sensitivity is a growing concept in the treatment of diabetic nephropathy. In the present study, MES+HS improved the whole-body insulin resistance in KKAy mice. Moreover, we previously showed that MES+HS directly activated Akt in podocytes in vivo and in vitro [28]. Akt is a critical downstream molecule of insulin signaling, and MES+HS was also reported to activate PI3K/Akt signaling pathway via enhanced tyrosine phosphorylation of insulin receptor [12]. Based on these findings, we hypothesized that MES+HS suppresses podocyte detachment and sequential podocyte loss by strengthening the structure of actin-rich foot process along with enhancing the insulin sensitivity of podocytes. Consistent with this concept, the expression of Synaptopodin, which is a functional marker of podocyte foot process, was significantly increased by MES+HS treatment. Also, it is possible that the increase of podocyte-associated gene expression by MES+HS is a secondary beneficial effect of the amelioration of some diabetic phenotypes.

In the present study, the voltage condition of MES we used was $5 \mathrm{~V}$, which is less than half of the intensity of the previous condition, which was $12 \mathrm{~V}$ [6]. For MES+HS-treated KKAy mice, $5 \mathrm{~V}$ means $2 \mathrm{~V} / \mathrm{cm}$ on average that is calculated by the distance of electrodes. Kondo et al. have reported that MES+HS improved metabolic abnormalities in T2DM patients with electro stimulation of $1.4 \pm 0.1 \mathrm{~V} / \mathrm{cm}$ [10]. Accordingly, we found beneficial effect in diabetic pathology and safety of long-term treatment of MES+HS using the condition that is closer to that used in clinical therapy. In conclusion, the present study shows that longterm MES+HS ameliorates hyperglycemia, insulin resistance and renal hypertrophy in KKAy mouse. These findings suggest that MES+HS 
has a potential to be a novel therapeutic and preventive approach for T2DM and diabetic nephropathy.

\section{Acknowledgments}

The device used for MES+HS treatment was kindly provided by the Tsuchiya Gum Co. Ltd. (Kumamoto, Japan).

\section{Funding information}

This work was supported by grants from the Ministry of Education, Science, Sports and Culture (MEXT) of Japan to H.K. (\#19390045) and by the Program for Leading Graduate Schools "HIGO (Health life science: Interdisciplinary and Glocal Oriented), MEXT, Japan.

\section{Conflict of Interest}

The authors declare no competing interests.

\section{Authors' contributions}

YK, YT, RF, KO, MP, YO, and KM performed the experiments and obtained the data. YK, MS and TS wrote the manuscript. TK, EA and HK designed and supervised the research. All authors approved the final version of the manuscript.

\section{References}

1. Gregg EW, Li Y, Wang J, Burrows NR, Ali MK, et al. (2014) Changes in diabetesrelated complications in the United States, 1990-2010. N Engl J Med 370: 1514-1523. [Crossref]

2. Skolnik NS, Ryan DH (2014) Pathophysiology, epidemiology, and assessment of obesity in adults. J Fam Pract 63: S3-3S10. [Crossref]

3. Bruce KD, Hanson MA (2010) The developmental origins, mechanisms, and implications of metabolic syndrome. J Nutr 140: 648-652. [Crossref]

4. Duran-Salgado MB, Rubio-Guerra AF1 (2014) Diabetic nephropathy and inflammation. World J Diabetes 5: 393-398. [Crossref]

5. Congdon NG, Friedman DS, Lietman T (2003) Important causes of visual impairment in the world today. JAMA 290: 2057-2060. [Crossref]

6. Morino S, Kondo T, Sasaki K, Adachi H, Suico MA, et al. (2008) Mild electrical stimulation with heat shock ameliorates insulin resistance via enhanced insulin signaling. PLoS One 3: e4068. [Crossref]

7. Fukuda R, Suico MA, Koyama K, Omachi K, Kai Y, et al. (2013) Mild electrical stimulation at $0.1-\mathrm{ms}$ pulse width induces $\mathrm{p} 53$ protein phosphorylation and $\mathrm{G} 2$ arrest in human epithelial cells. J Biol Chem 288: 16117-16126. [Crossref]

8. Matsuyama S, Moriuchi M, Suico MA, Yano S, Morino-Koga S, et al. (2014) Mild electrical stimulation increases stress resistance and suppresses fat accumulation via activation of LKB1-AMPK signaling pathway in C. elegans. PLoS One 9: e114690. [Crossref]

9. Morino S, Suico MA, Kondo T, Sekimoto E, Yano S, et al. (2008) Mild electrical stimulation increases ubiquitinated proteins and Hsp72 in A549 cells via attenuation of proteasomal degradation. J Pharmacol Sci 108: 222-226. [Crossref]
10. Kondo T, Ono K, Kitano S, Matsuyama R, Goto R, et al. (2014) Mild Electrical Stimulation with Heat Shock Reduces Visceral Adiposity and Improves Metabolic Abnormalities in Subjects with Metabolic Syndrome or Type 2 Diabetes: Randomized Crossover Trials. EBio Medicine 1: 80-89. [Crossref]

11. Kondo T, Motoshima H, Igata M, Kawashima J, Matsumura T, et al. (2014) The role of heat shock response in insulin resistance and diabetes. Diabetes Metab J 38: 100-106. [Crossref]

12. Morino-Koga S, Yano S, Kondo T, Shimauchi Y, Matsuyama S, et al. (2013) Insulin receptor activation through its accumulation in lipid rafts by mild electrical stress. J Cell Physiol 228: 439-446. [Crossref]

13. Kennedy AJ, Ellacott KL, King VL, Hasty AH (2010) Mouse models of the metabolic syndrome. Dis Model Mech 3: 156-166. [Crossref]

14. O'Brien SP, Smith M, Ling H, Phillips L, Weber W, et al. (2013) Glomerulopathy in the KK.Cg-A(y) /J mouse reflects the pathology of diabetic nephropathy. $J$ Diabetes Res 2013: 498925.

15. Fukuda R, Suico MA, Kai Y, Omachi K, Motomura K, et al. (2015) Podocyte p53 Limits the Severity of Experimental Alport Syndrome. J Am Soc Nephrol. [Crossref]

16. Iwatsuka H, Shino A, Suzuoki Z (1970) General survey of diabetic features of yellow KK mice. Endocrinol Jpn 17: 23-35. [Crossref]

17. Nakamura M, Yamada K (1967) Studies on a diabetic (KK) strain of the mouse. Diabetologia 3: 212-221. [Crossref]

18. Baumgartl HJ, Sigl G, Banholzer P, Haslbeck M, Standl E (1998) On the prognosis of IDDM patients with large kidneys. Nephrol Dial Transplant 13: 630-634. [Crossref]

19. Wolf G, Ziyadeh FN (1999) Molecular mechanisms of diabetic renal hypertrophy. Kidney Int 56: 393-405. [Crossref]

20. Li JJ, Kwak SJ, Jung DS, Kim JJ, Yoo TH, et al. (2007) Podocyte biology in diabetic nephropathy. Kidney Int Suppl : S36-42. [Crossref]

21. Manda G, Checherita AI, Comanescu MV, Hinescu ME (2015) Redox Signaling in Diabetic Nephropathy: Hypertrophy versus Death Choices in Mesangial Cells and Podocytes. Mediators Inflamm 2015: 604208.

22. Shankland SJ (2006) The podocyte's response to injury: role in proteinuria and glomerulosclerosis. Kidney Int 69: 2131-2147. [Crossref]

23. Coward RJ, Welsh GI, Yang J, Tasman C, Lennon R, et al. (2005) The human glomerular podocyte is a novel target for insulin action. Diabetes 54: 3095-3102. [Crossref]

24. Wolf G, Chen S, Ziyadeh FN (2005) From the periphery of the glomerular capillary wall toward the center of disease: podocyte injury comes of age in diabetic nephropathy. Diabetes 54: 1626-1634. [Crossref]

25. Kondo T, Sasaki K, Adachi H, Nakayama Y, Hatemura M, et al. (2010) Heat shock treatment with mild electrical stimulation safely reduced inflammatory markers in healthy male subjects. Obes Res Clin Pract 4: e83-e162.

26. Sun YB, Qu X, Howard V, Dai L, Jiang X, et al. (2015) Smad3 deficiency protects mice from obesity-induced podocyte injury that precedes insulin resistance. Kidney Int 88: 286-298. [Crossref]

27. Welsh GI, Hale LJ, Eremina V, Jeansson M, Maezawa Y, et al. (2010) Insulin signaling to the glomerular podocyte is critical for normal kidney function. Cell Metab 12: 329340. [Crossref]

28. Koga T, Kai Y, Fukuda R, Morino-Koga S, Suico MA, et al. (2012) Mild electrical stimulation and heat shock ameliorates progressive proteinuria and renal inflammation in mouse model of Alport syndrome. PLoS One 7: e43852. [Crossref]

Copyright: (C2015 Kai Y. This is an open-access article distributed under the terms of the Creative Commons Attribution License, which permits unrestricted use, distribution, and reproduction in any medium, provided the original author and source are credited. 